\title{
Rehydration with fructose worsens dehydration-induced renal damage
}

\author{
Tamara Milagres ${ }^{1}$, Fernando E. García-Arroyo², Miguel A. Lanaspa', Gabriela Garcia', Takuji Ishimoto', \\ Ana Andres-Hernando', Masanari Kuwabara', Thomas Jensen'1, Yuka Sato', Jason Glaser ${ }^{3}$, \\ Laura G. Sánchez-Lozada², Richard J. Johnson' (1) and Carlos Roncal-Jimenez ${ }^{1 *}$
}

\begin{abstract}
Background: Increasing evidence suggests heat stress induced chronic kidney disease (CKD) may be mediated by endogenous fructose generation and may be exacerbated by rehydration by fructose-containing solutions. We have recently reported a model of CKD induced by heat stress. Here we test the hypothesis that rehydration with fructose may induce worse kidney injury than rehydration with equal amounts of water, and we also test if this fructose-induced injury is associated with activation of inflammasomes in the kidney.

Methods: Mice were recurrently exposed to heat $\left(39.5 C^{0}\right.$ for $30 \mathrm{~min} / \mathrm{h}, 5$ times daily for 5 wks) with rehydration consisting of $6 \mathrm{ml}$ each night of water (Heat, $n=7$ ) or fructose (Heat+F, 10\%, n=7), and were compared to control mice on water (Control, $n=7$ ) or fructose (Fructose, $n=7$ ). Various markers of renal injury were assessed.

Results: Compared to control animals, there was a progressive worsening of renal injury (inflammation and fibrosis) with fructose alone, heat stress alone, and heat stress with fructose rehydration $(P<0.01$ by ANOVA). The combination of heat stress with rehydration with fructose was associated with increased intrarenal expression of the inflammasome markers, NLRP3 and IL-18, compared to heat stress alone. In addition, heat stress with or without fructose was associated with increased expression of caspase -3 and monocyte chemoattractant protein-1 levels. Fructose administration was also associated with an increase in serum copeptin levels (a biomarker of vasopressin) and elevated copeptin was also observed in mice undergoing heat stress alone.

Conclusions: These studies suggest that heat stress may activate intrarenal inflammasomes leading to inflammation and renal injury, and provide evidence that rehydration with fructose may accelerate the renal injury and inflammatory response.
\end{abstract}

Keywords: Fructose, Mesoamerican nephropathy, Heat stress, Vasopressin

\section{Background}

Heat stroke is a concern throughout the world, not only as a complication of exercise (such as marathon running) and of military campaigns, but also for individuals working outside in both rural settings and in hot urban environments [1-6]. Epidemics of heat stroke associated with extreme heat events (heat waves) were reported in Chicago in 1995 and Europe in 2003 [1, 7-10]. Indeed, heat stroke is likely to become more common as

\footnotetext{
* Correspondence: Carlos.Roncal@ucdenver.edu

${ }^{1}$ Division of Renal Diseases and Hypertension, Nephrology Division, Mail Stop C281, University of Colorado Anschutz Medical Campus, 12700 East 19th Ave 7th Floor Offices, Aurora, CO 80045, USA

Full list of author information is available at the end of the article
}

temperatures rise, for now nearly $75 \%$ of heat extremes can be attributed to climate change [11, 12].

Most emphasis on heat stroke relates to the acute risk for multi-organ failure and death. However, heat stroke has also been associated with residual liver and kidney damage [13, 14]. More recently, we have raised the hypothesis that chronic recurrent heat stress might be able to induce chronic kidney disease (CKD), and we have hypothesized this may be of relevance to the epidemics of CKD being observed in among agricultural workers in various hot communities in Central America, India, Sri Lanka and elsewhere [15-17].

To investigate this hypothesis, we developed a model of CKD induced in mice by repetitive heat stress and

(c) The Author(s). 2018 Open Access This article is distributed under the terms of the Creative Commons Attribution 4.0 International License (http://creativecommons.org/licenses/by/4.0/), which permits unrestricted use, distribution, and reproduction in any medium, provided you give appropriate credit to the original author(s) and the source, provide a link to the Creative Commons license, and indicate if changes were made. The Creative Commons Public Domain Dedication waiver (http://creativecommons.org/publicdomain/zero/1.0/) applies to the data made available in this article, unless otherwise stated. 
dehydration over a 5 week period. Using this model, we found that if mice were hydrated during the heat stress period, they did not develop renal injury, whereas if they hydrated after the heat/dehydration period, that they developed mild tubulointerstitial kidney disease [18]. In this same study we found evidence that heat stress/dehydration caused activation of aldose reductase in the kidney, an osmotically-sensitive enzyme that converts glucose to sorbitol, which is then further metabolized in the renal cortex to fructose. Fructose is known to be metabolized in the proximal tubule by the enzyme fructokinase, and this results in a drop in ATP levels, the generation of uric acid, and a burst of oxidative stress that can cause tubular injury [19]. Indeed, mice lacking fructokinase were protected from the renal injury of recurrent heat stress [18].

Given that many individuals working in the rural communities hydrate themselves with sugary beverages, including soft drinks and fruit juices, one might hypothesize that rehydration with fructose-containing solutions might exacerbate our model of renal injury of heat stress and dehydration. In this regard, recently Garcia-Arroyo et al. developed a model of mild heat stress and dehydration in rats in which rats were exposed for only $1 \mathrm{~h}$ a day to heat $\left(36^{\circ} \mathrm{C}\right)$ followed by $2 \mathrm{~h}$ rehydration with water or a soft drink mixture containing $11 \%$ fructose-glucose mixture [20]. This mild model of dehydration does not result in chronic interstitial fibrosis but does manifest with tubular injury and intrarenal oxidative stress [20]. Importantly, this study found that rehydration with a sugary beverage resulted in worse tubular injury and oxidative stress.

We therefore tested the hypothesis that fructose might accelerate heat stress induced CKD in our model. Furthermore, recently it has been recognized that subjects developing CKD in Central America often have evidence of intermittent acute renal injury with a predominance of inflammatory cells on their biopsy [17, 21]. This was viewed as counter to the generally held idea that the acute renal injury might be more similar to acute tubular necrosis. However, heat stroke is also associated with a pronounced renal inflammatory infiltrate [17]. We thus investigated if heat stress might be associated with an increase in inflammasome-associated proteins, and tested the hypothesis that this might be worsened by fructose ingestion.

\section{Methods}

\section{Animals}

Male 8-week old C57BL/6 J wild type mice (WT, Jackson Lab, Bar Harbor, ME) were maintained in temperatureand humidity-controlled specific pathogen-free conditions on a 14-h dark/10-h light cycle and fed regular diet ad libitum (Harlan Teklad; no. 2918, containing 58\% carbohydrate, $24 \%$ protein, and $18 \%$ fat), with free access to tap water.

\section{Experimental design}

The experimental study consisted of four groups $(n=7$ each): WT control (Control); WT fructose (Fructose); WT mice exposed to heat and water restriction with water rehydration (Heat); and WT mice exposed to heat and water restriction with fructose-containing drinking water (10\% fructose) as the rehydration fluid (Heat $+\mathbf{F})$. The heat stress-dehydration protocol has been previously reported and consisted of placement of mice in a heat chamber set at $39.5^{\circ}$ Celsius for $30 \mathrm{~min}$ per hour over $8 \mathrm{~h}$ (7 episodes/day), 5 days/week, for 5 weeks [18]. In between heat periods mice were allowed to rest at room temperature. Fluids were restricted during the heat/dehydration period, and then $6 \mathrm{ml}$ of rehydration fluid per day were provided per mouse afterwards with each mouse housed in its own cage using millimetric water bottles, consisting of water in the Heat group and fructose water in the Heat $+\mathbf{F}$ group. This degree of rehydration fluid was calculated based on the overall $24 \mathrm{~h}$ fluid intake required by mice of this age and weight in our past study [18]. Mice were sacrificed two hours after the last cycle of heat stress (prior to rehydration) at the end of the 5th week by anesthesia (isoflurane) with cardiac exsanguination and collection of serum, puncture of the bladder for collection of urine, and removal of kidney tissues for analyses.

All experiment were conducted with adherence to the National Institute of Health Guide for the Care and Use of Laboratory Animals. The animal protocol was approved by the Animal Care and Use Committee of the University of Colorado.

\section{Biochemical analyses}

Urine was collected at the end of the study from the bladder and were analyzed for urine osmolality using the Freezing-Point Osmometry method (Advance Instruments Micro Osmometer- Norwood Massachusetts USA). Serum and urine creatinine concentrations were analyzed with the high-performance liquid chromatography-tandem mass spectrometry method [22]. Urinary albumin was measured using a Colorimetric Albuwell Assay Kit (Exocell Co., PA). Serum copeptin levels were measured using Elisa enzyme- linked immunosorbent Assay kit for copeptin (Cloud-Clone Corp., Houston TX) Serum fructose was measured using the EnzyChrom Fructose Assay Kit (Bioassay Systems, Hayward, CA) and serum uric acid was measured using QuantiChrom Uric Acid assay kit (BioAssay Systems).

Kidney tissue samples were homogenized in a buffer containing $2 \mathrm{mM} \mathrm{MgCl}_{2}, 1 \mathrm{mM}$ EGTA, $1 \mathrm{mM}$ DTT, and $0.5 \% \quad(\mathrm{vol} / \mathrm{vol})$ Triton X-100. Homogenates were 
centrifuged at 13,000 rpm for $10 \mathrm{~min}\left(4{ }^{\circ} \mathrm{C}\right)$ and protein in the collected supernatant quantified (BCA protein assay Kit - Pierce, Rockford, IL). Renal fructose and uric acid levels were assessed by utilizing the Bioassay Systems kits (see above) and values normalized to protein lysate concentration. Renal monocyte chemoattractant protein (MCP-1) was measured on cortical lysates by Mouse MCP-1 ELISA Kit (Invitrogen ThermoFisher Grand Island NY-USA) and corrected for total protein (Pierce, BCA protein assay Rockford, IL-USA).

\section{Histology}

Kidney tissues were fixed in $10 \%$ formalin or methyl Carnoy's and embedded in paraffin. Three micron axial sections of the kidney were stained with periodic acid-Schiff reagent (PAS). Proximal tubular brush border loss was assessed by immunostaining for angiotensinconverting enzyme (ACE) using antimurine ACE antibody; ( $\& D$, Minneapolis, $M N)$. Renal fibrosis was determined by immunohistochemical staining for type III collagen with a goat anti-type III collagen antibody (Southern Biotech, Birmingham-AL-USA). Macrophage infiltration were detected using Rat Anti-Mouse F4/80 antibody (Serotec, Oxford, UK). The number of positive cells for F4/80 was counted using an Aperio scanner (Aperio Technologies, Vista, CA). Additionally, we evaluated the inflammatory response by immunostaining for Nod-like receptor family-3 (NLPR3) with rabbit anti-mouse NRLRP3 (Novus Biologicals, Littleton CO) and interleukin 18 (IL-18) using rabbit polyclonal anti-IL-18 antibody (Abcam, Cambridge MA). Caspase-3, involved in apoptosis, as detected using a rabbit polyclonal anti-caspase 3 antibody (Abcam). The software allows color recognition and positive cells were identified as \% positive color saturation at 20 magnification in a blinded manner using at least 15 fields for each biopsy sample. For the fibrosis and ACE staining, digital images at 20X magnification of approximately 10 cortical fields were analyzed using Image scope of Aperio Scanner software. The percent positive area was determined as the 3,3-diaminobenzidine-positive pixel values per negative pixel values in each section.

\section{Validation study}

We also determined whether inflammasomes and apoptosis-related pathways might be stimulated in the kidney in another model of heat stress and whether this response would be amplified by rehydration with fructose-containing drinks versus water. Specifically, we obtained tissues from male Wistar rats that had been exposed daily ( 5 times/week for 4 weeks) to one hour of heat stress $\left(37{ }^{\circ} \mathrm{C}\right.$ for one hour) followed by two hours ad libitum hydration with either water or a $10 \%$ fructose solution. The rest of the day the rats had regular water and normal rat chow ad libitum. This model has previously been shown to result in low grade tubular injury and renal oxidative stress that was worse in the fructose-rehydrated rats [23, 24]. We used renal tissues from one of these studies [24] to evaluate for the presence of NLRP3, caspase-3 and IL-1 $\beta$ by western blot [24] using specific primary antibodies (anti-NLRP3 antibody (ab4207, Abcam), anti-Cleaved Caspase 3 (Asp 175) antibody (Cell Signaling), and anti-IL-1 $\beta$ (C-20, Santa Cruz Biotechnology), with loading controlled using an anti-beta actin antibody (Genetex).

\section{Statistical analysis}

Statistical analyses were performed using the GraphPad Prism version 6 (GraphPad Software, Inc. La Jolla, CA). All data are presented as the mean \pm s.e.m. Independent replicates for each data point (n) are identified in figure legends and one-way analysis of variance (ANOVA) with the Bonferroni post hoc test used for individual comparisons. We also used the Student's t-test to specifically compare heat alone (Heat) to heat plus fructose (Heat + F) groups since the experiment was designed specifically for this comparison (see comments in limitation section). $P<0.05$ was regarded as statistically significant.

\section{Results}

\section{General findings}

Recurrent heat stress and dehydration were induced in two groups of mice over a 5 week period in which the only difference was that one group received water rehydration (Heat) and the other group received the same amount of drinking water containing fructose $(10 \%)$ $($ Heat $+\mathrm{F})$. We also studied two groups of mice not exposed to heat stress/dehydration administered the same dose of water or fructose-containing water. While each day the mice undergoing heat stress and dehydration would lose significant weight $(14.2 \pm 0.8$ vs 13.8 $\pm 1.2 \%$ in the Heat vs Heat $+\mathrm{F}$ groups, $\mathrm{p}=\mathrm{NS}$ ), they were able to fully rehydrate at night and no mortality was observed. At the end of the study there were significant differences in weight by one way ANOVA, with the fructose control showing higher weight compared with the heat stress/dehydration group $(p=0.003)$ and heat stress/dehydration groups plus fructose group $(p<$ 0.001 ), but with no differences between the other two groups (Table 1).

\section{Effect on dehydration markers}

Both groups of mice exposed to heat stress and dehydration showed increases in serum osmolarity, and serum vasopressin (determined by measuring serum copeptin) levels (Table 1). Serum copeptin levels increased more in the fructose rehydrated control group compared to the water rehydrated group (Table 1), consistent with studies 
Table 1 General Characteristics of Experimental Groups

\begin{tabular}{llllll}
\hline Parameters & Control & Fructose & Heat & Heat+F & Anova $p$ values \\
\hline Body weight Basal $(\mathrm{g})$ & $29.5 \pm 2.1$ & $29.3 \pm 2.0$ & $30.0 \pm 2.3$ & $30.2 \pm 2.1$ & NS \\
Body weight After $5 \mathrm{~W}(\mathrm{~g})$ & $31.3 \pm 3.6$ & $33.3 \pm 2.1$ & $28.8 \pm 1.9$ & $28.3 \pm 1.2$ & $<0.001$ \\
Serum Osmolality (mOsm/Kg) & $307 \pm 6.1$ & $312.2 \pm 6$ & $343.6 \pm 16.8$ & $340 \pm 29.8$ & $<0.0001$ \\
Serum Copeptin $(\mathrm{pg} / \mathrm{ml})$ & $10 \pm 3.9$ & $25.3 \pm 1.4$ & $18.4 \pm 4.5$ & $25 \pm 4.2$ & $<0.0001$ \\
\hline
\end{tabular}

Comparisons: The Fructose control group has a higher body weight at the end of the study compared to HEAT $(p<0.01)$ and HEAT+F groups $(p<0.001)$ by Bonferroni test. Controls have slower serum osmolality than HEAT $(p<0.001)$ and HEAT+F groups $(p<0.01)$ by Bonferroni. The Fructose group has lower serum osmolality than HEAT $(p<0.05)$. The Controls have lower serum copeptin than Fructose $(p<0.001)$, HEAT $(p<0.01)$ and HEAT+F groups $(p<0.001)$ by Bonferroni test. The Fructose group has higher copeptin levels than HEAT $(p<0.05)$. The HEAT group tends to have lower copeptin levels than the HEAT+F group $(p=0.08)$ by Bonferroni, and is significant by t-test $(p=0.049)$

suggesting fructose can stimulate vasopressin release $[25,26]$. The effect of fructose to increase serum copeptin was also greater in the Heat $+\mathrm{F}$ compared to the Heat alone group when analyzed by Student's t-test $(p=0.049)$.

\section{Effect on renal function}

Serum creatinine was elevated in both heat stress groups compared to the control and fructose alone groups (Table 2). Also, the groups exposed to heat stress and dehydration plus fructose had a tendency for elevated albuminuria compared to the control group $(P=0.055)$ or fructose group $(P=0.051)$ by Bonferroni post hoc test. However, no significant differences were noted between the heat stress and heat stress plus fructose group.

\section{Effect on renal histology}

Compared to controls animals, mice exposed to heat stress and dehydration (HEAT) and $($ HEAT + F) showed evidence for focal proximal tubular injury (Fig. 1) with loss of proximal tubular brush border as noted by immunostaining for angiotensin converting enzyme (ACE) (Fig. 2a-c, Table 2). The mice receiving fructose as rehydration $(\mathrm{HEAT}+\mathrm{F})$ showed more severe loss of proximal tubule brush border compared to heat alone (HEAT) $(P<0.001)$ (Table 2$)$. This was associated with interstitial macrophage infiltration (F4/80 positive cells) (Fig. 2d-f, Table 2). Interstitial fibrosis (noted by collagen
III staining) was also increased in the renal cortex of heat stressed mice, but there was no difference between HEAT and HEAT+F groups (Table 2, Fig. 2g-i).

\section{Effect on sorbitol, fructose and uric acid levels in the renal cortex}

The polyol (aldose reductase) pathway has been reported to be induced in the renal cortex of this heat-stressed model in mice [18]. Sorbitol, the product of aldose reductase, was elevated in both groups of mice exposed to heat stress and tended to be higher in the fructose treated group $(\mathrm{HEAT}+\mathrm{F})$ compared to heat stress alone (HEAT), although this was not significant (Fig. 3a). We also measured fructose levels in the renal cortex. Surprisingly, fructose levels in renal cortex were not higher in the heat stress group compared to the control group and other groups (Fig. 3b). Fructose is metabolized in the proximal tubule by fructokinase with the generation of uric acid [19]. Uric acid levels were higher in the renal cortex of heat stress and fructose $(\mathrm{HEAT}+\mathrm{F})$ group compared to control group (Fig. 3c).

We have previously reported that fructose generated uric acid can stimulate MCP-1 production in proximal tubular cells [19, 27]. Soluble uric acid has also been reported to stimulate inflammasome responses in tubular cells [28]. We therefore measured the renal cortical content of the chemokine, MCP-1, (Fig. 3d) as well as markers of the inflammasome, including NRLP3

Table 2 Effect of Fructose with or without Heat on Renal Disease

\begin{tabular}{|c|c|c|c|c|c|}
\hline Parameters & Control & Fructose & Heat & Heat+F & Anova $p$ values \\
\hline Serum Creatinine $(\mu \mathrm{g} / \mathrm{ml})$ & $0.38 \pm 0.1$ & $0.36 \pm 0.1$ & $0.69 \pm 0.2$ & $0.72 \pm 0.2$ & $P<0.001$ \\
\hline Urine Albumin ( $\mu \mathrm{g} / \mathrm{mg}$ of $\mathrm{Cr}$ ) & $26.1 \pm 5.1$ & $27.5 \pm 7.5$ & $54.3 \pm 28.1$ & $65.5 \pm 32.3$ & $P<0.05$ \\
\hline ACE Positivity \% (proximal tubular brush border integrity) & $10.3 \pm 2.3$ & $6.6 \pm 1.8$ & $6.9 \pm 1.6$ & $4.5 \pm 3.1$ & $P<0.0001$ \\
\hline Monocyte Accumulation (F4/80) \% & $0.13 \pm 0.3$ & $0.13 \pm 0.2$ & $0.25 \pm 0.3$ & $0.55 \pm 0.6$ & $P<0.05$ \\
\hline Interstitial Fibrosis (Coll-III) \% & $0.23 \pm 0.1$ & $0.28 \pm 0.3$ & $0.43 \pm 0.3$ & $0.53 \pm 0.4$ & $P<0.0001$ \\
\hline
\end{tabular}

Comparisons: The serum creatinine of Control is lower than HEAT $(P<0.05)$ and HEAT+F group $(P<0.05)$ by Bonferroni. Fructose has lower serum creatinine than HEAT $(P<0.05)$ and HEAT+F $(P<0.05)$. Controls tends to have lower urinary albumin than HEAT+F $(P=0.055)$ and Fructose tends to be lower than HEAT+F $(P=0.051)$ by Bonferroni. Controls have more proximal tubular brush border (ACE positivity) than Fructose $(P<0.001)$, Heat $(P<0.001)$ and HEAT+F $(P<0.001)$ by Bonferroni. The Fructose group has more brush border (ACE positivity) than HEAT+F $(P<0.01)$. The HEAT group has more ACE positivity than HEAT+F group by $\mathrm{t}$ test $(P<0.001)$. Controls have significantly less interstitial macrophage accumulation than HEAT+F $(P<0.05)$ by Bonferroni. Controls have significantly less interstitial fibrosis than HEAT $(P<0.01)$ and HEAT $+\mathrm{F}(P<0.001)$ by Bonferroni post hoc test 

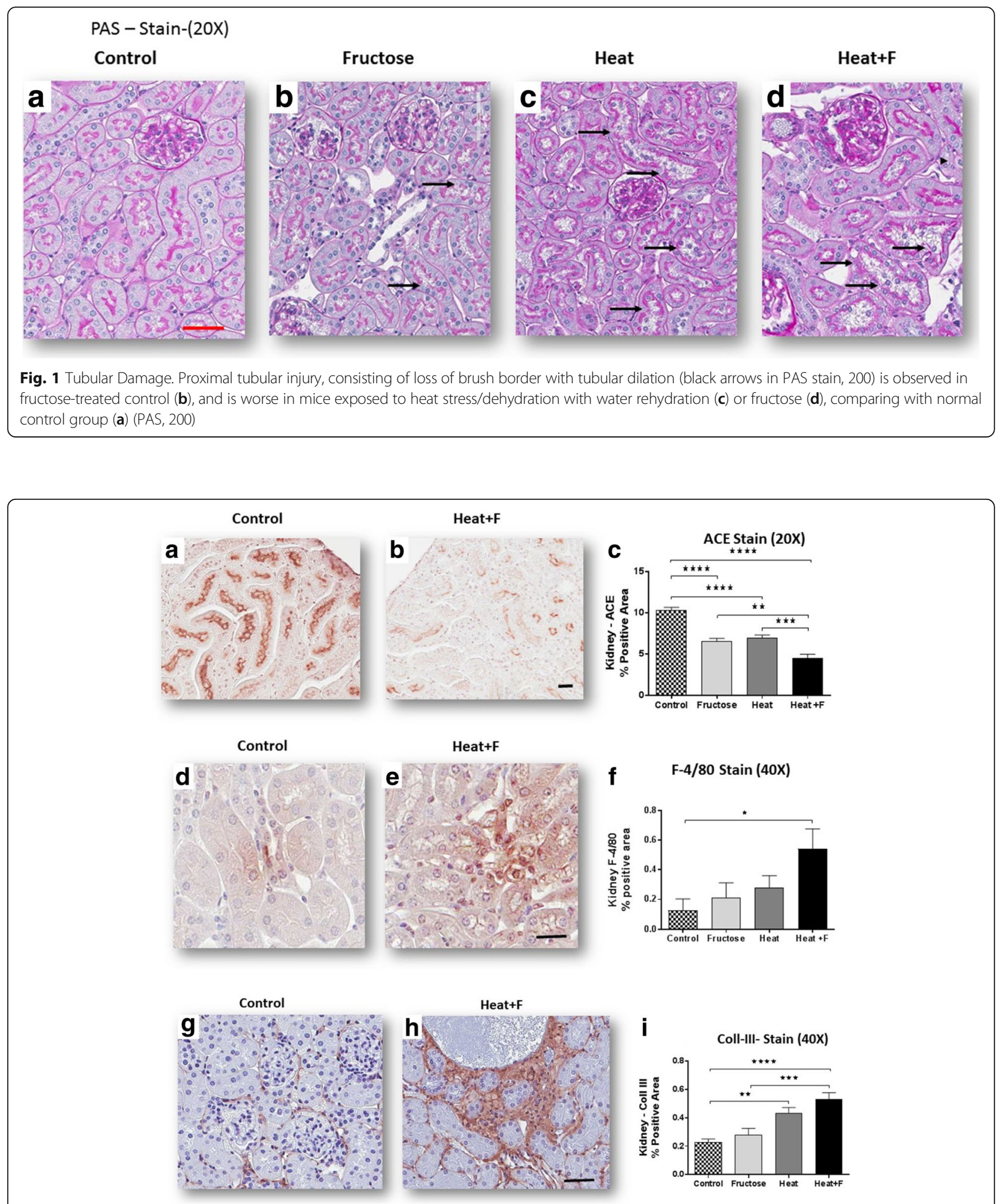

Fig. 2 Renal injury. Proximal tubular injury was assessed by staining for proximal tubular brush border for ACE (a-c, 200), interstitial inflammation was determined by staining for F4/80 positive macrophages (d-f, 400X), and interstitial fibrosis by staining for type III collagen (g-i, 200). Heat stress was associated with loss of proximal tubular brush border, interstitial inflammation and interstitial fibrosis which tended to be worse with fructose rehydration. Scale bar $50 \mu \mathrm{m}$. Statistical analysis was performed using ANOVA with Bonferroni correction for individual comparisons. Key: ${ }^{*}, p<0.05 ;{ }^{* *}, p<0.01 ;{ }^{* * *} p<0.001,{ }^{* * * *}, p<0.0001$ 

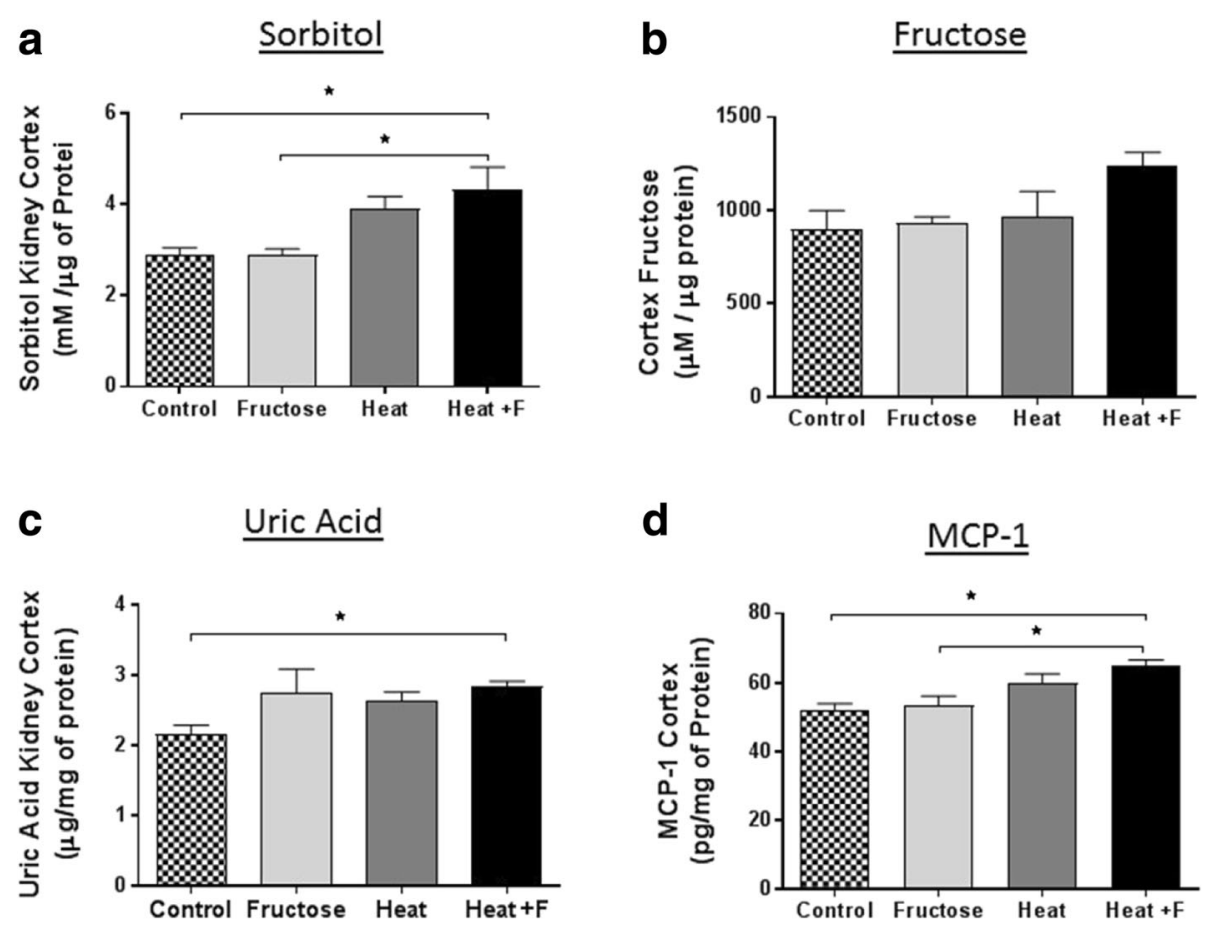

Fig. 3 Renal Cortex Sorbitol, Fructose and Uric Acid Levels. a Sorbitol levels were increased in heat plus fructose (HEAT+F) exposed mice compared to wild type controls by Bonferroni post hoc test $(p=0.013)$. The Fructose control group also had lower levels of sorbitol than the HEAT+F group $(p=0.015)$. $\mathbf{b}$ There was no significant differences of fructose in renal cortex for individual group comparisons by Bonferroni post hoc test. $\mathbf{c}$ HEAT+F group had higher uric acid in renal cortex compared to the control by Bonferroni post hoc test $(p=0.042)$. $\mathbf{d}$ HEAT+F group had higher MCP-1 in renal cortex than control group $(P=0.013)$ and fructose group $(P=0.016)$ by Bonferroni post hoc test. Key: ${ }^{*} p<0.05$

(Fig. 4a-c); Caspase 3 (Fig. 4d-f), and the macrophage product, IL-18 (Fig. 4g-i). Specifically, NLPR3, caspase 3 and IL-18, and all were higher in the mice with heat stress that were rehydrated with fructose as opposed to water, with NLRP3 and IL-18 levels were significantly higher in the $\mathrm{HEAT}+\mathrm{F}$ group compared to HEAT alone.

\section{Validation study}

We also performed additional analyses on another model of heat stress induced renal injury to determine if fructose rehydration was associated with an induction of inflammasome markers in that model. Specifically, we have reported that Wistar rats exposed to transient heat stress $\left(1 \mathrm{~h}\right.$ daily of $37^{\circ} \mathrm{C}$ ) will develop mild tubular injury and renal oxidative stress that is worse if they are rehydrated following the heat stress with fructose-containing solutions as opposed to water [23, 24]. We obtained some of the rat tissues from one of these prior studies and probed the tissue for markers of inflammasomes and apoptosis by Western blot. As shown in Fig. 5, fructose rehydration was associated with significantly greater expression of the inflammasome markers NLRP3, caspase- 3 , and interleukin $1 \beta$.

\section{Discussion}

Heat Stress and dehydration are well known to cause a 'prerenal' type of kidney injury, but it has historically been considered to not be associated with tubular injury and to be fully reversible with hydration. Recently some have proposed that recurrent heat stress and dehydration may be a cause of CKD, based primarily on epidemiological studies performed on sugarcane workers who are developing CKD in Central America [29-32]. To explore this possibility, our group developed a model of CKD in mice induced by repetitive exposure to heat stress over a 5 week period [18]. These mice developed chronic tubulointerstitial inflammation and interstitial fibrosis, both which are characteristic of the CKD in Central America (Mesoamerican Nephropathy) [33].

Here we evaluated the effect of equal hydration with fructose containing water or regular water in our chronic model of heat stress. The primary finding was that fructose worsened the renal tubular injury (as noted by ACE staining) and resulted in marked tubulointerstitial inflammation and a tendency for worse renal fibrosis (although the latter two findings were not significant). In addition, we found evidence for an increase in inflammatory pathways in the renal cortex, including the chemokine MCP-1, inflammasome, and apoptosis markers 

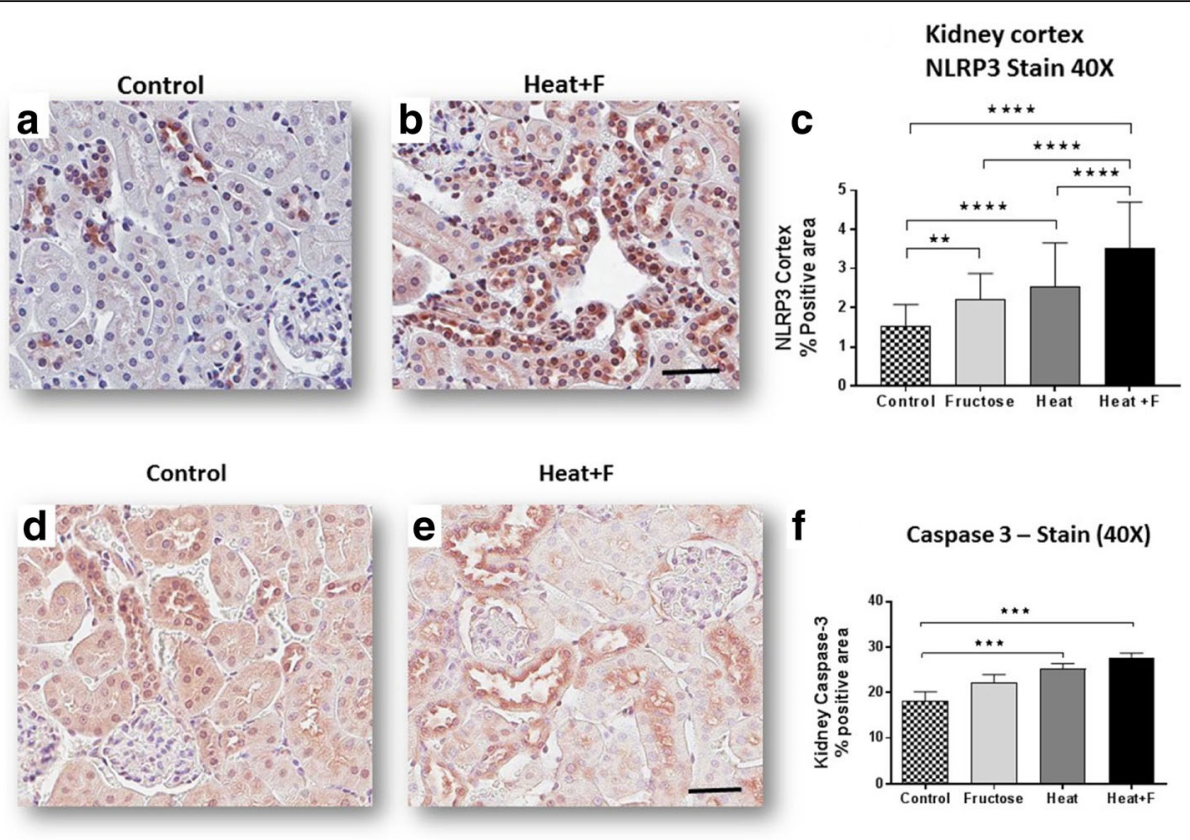

Caspase 3 - Stain (40x)
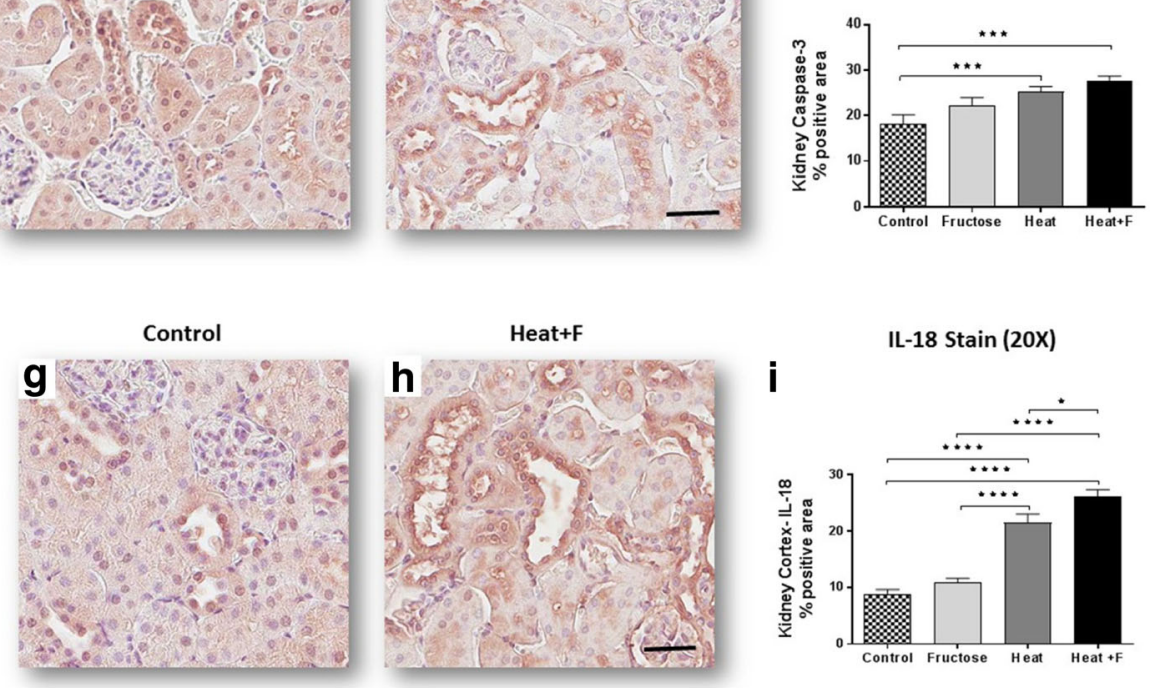

Fig. 4 Inflammatory response and renal injury. Renal cortical NLRP3 expression showed stepwise increase with the highest level in the HEAT+F group by quantitation of \% NLRP3 positive stain area (a-c, 400x, Scale bar $50 \mu \mathrm{m})$. Caspase 3 expression was higher in heat stress + Fructose (HEAT+F) group compared to the other groups (d-f, 400× Scale bar $50 \mu \mathrm{m})$. Similar findings were shown for Interleukin 18 (g-i, $400 \times$, Scale bar $50 \mu \mathrm{m})$. Key: ${ }^{*}, p<0.05 ;{ }^{* *}, p<0.01 i^{* * *} p<0.001,{ }^{* * *}, p<0.0001$

(NLRP3, caspase 3 and IL-18). Thus, dietary fructose should be viewed as a potential amplifier of the renal injury process, and the mechanism is tightly linked with stimulation of inflammatory processes.

While our model is in mice, we have also developed a model of heat stress associated renal injury in rats $[23,24]$. This is a milder model in which rats are exposed to only one hour of heat stress, and rats develop tubular injury without fibrosis associated with renal oxidative stress and systemic inflammation. In this model we have also shown that rehydration with fructose can result in greater renal oxidative stress than hydration with water alone [23, 24]. Since one of the new findings in this study was the finding that fructose might increase the expression of inflammasome markers, we tested kidney tissues from this previously published rat model [24] and confirmed that rehydration with fructose significantly increases the expression of inflammasome and apoptosis markers (NLRP3, caspase 3, and IL-1 $\beta$ ) compared to water hydration alone.

The mechanisms by which repetitive heat stress may cause kidney damage has been a 'hot' topic. Some studies suggest a role for vasopressin [24, 34], which has long been known to induce some tubular and glomerular injury [35]. Indeed, in the rat model of heat stress associated renal injury, conivaptan, which blocks V1a and V2 receptors, could reduce renal injury associated with recurrent heat stress [24]. We have also reported that stimulation of V2 receptors with desmopressin can accelerate injury in our mouse model [34]. An interaction between fructose and vasopressin is also likely, as we have reported that endogenous fructose (produced in the hypothalamus in dehydration through an osmolality activation of aldose reductase) can stimulate vasopressin synthesis [26], and dietary fructose has also been 

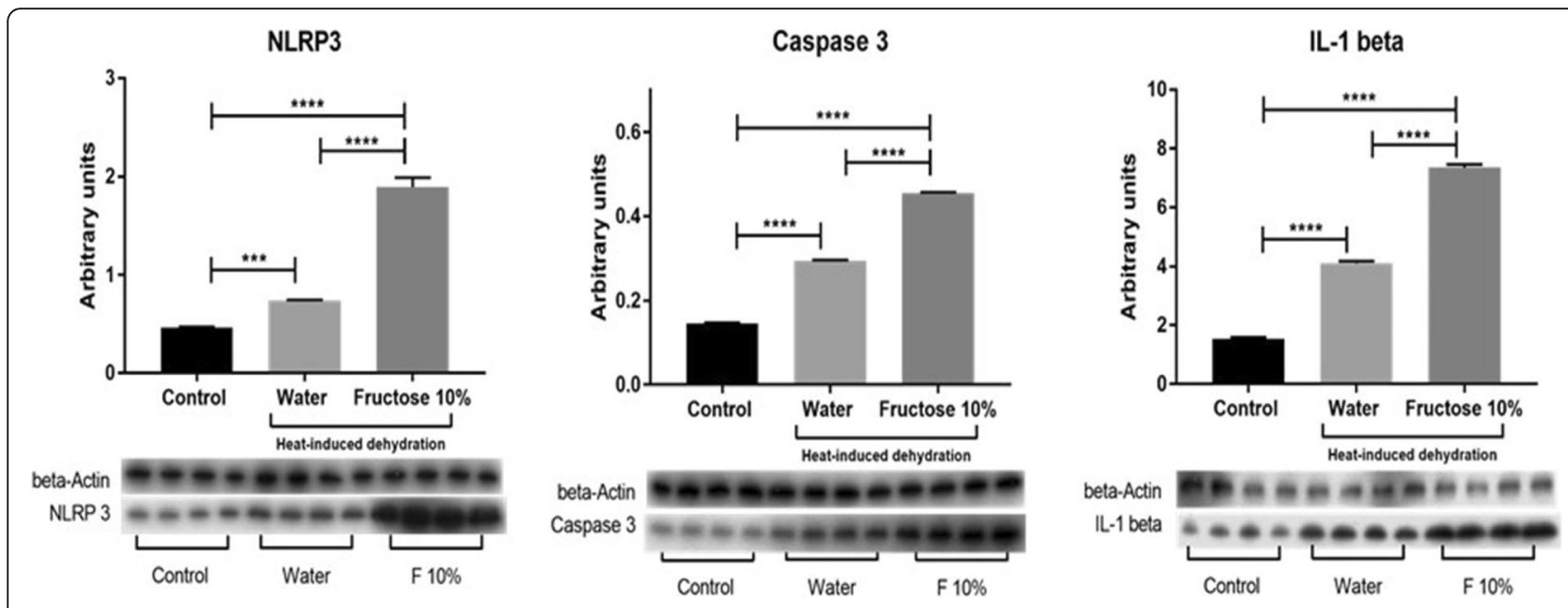

Fig. 5 Inflammasome Activation in a Rat Model of Heat Stress. Protein lysates from the kidneys of a rat model of heat stress [24] were used to confirm whether rehydration with fructose containing solutions can increase the expression for inflammasome and apoptosis markers in the kidney. Heat stress with water rehydration could induce an increase in NRLP3, Caspase-3, and IL-1 $\beta$ protein in renal tissues compared to normal controls. However, when rats were rehydrated with fructose (10\% in the drinking water), the expression of these inflammasome markers increased markedly and were significantly greater than rehydration with water alone. $N=4$ per group

reported to increase circulating vasopressin levels [23-25]. We also noted an increase in serum copeptin in our fructose hydrated mice. Indeed, mice that cannot metabolize fructose easily (fructokinase knockout) appear to be protected from heat stress induced renal disease [18]. Similarly, conivaptan treatment could block renal injury in the rat model of heat stress, especially in animals rehydrated with fructose [23]. A prime question resulting from this work is whether sports drinks and rehydration solutions that are being used to hydrate individuals in hot environments may be increasing their risk for renal damage.

A weakness in our study was that the renal fibrosis observed in our fructose-rehydrated mice was not significantly worse than heat stress alone, and as such we cannot conclude that fructose definitely worsens heat stress nephropathy. However, fructose hydration was associated with significantly worse tubular injury and expression of inflammatory markers. It is possible that a difference would have been shown if the study had been conducted over a longer period. Nevertheless, the overall trend is suggestive that fructose, and perhaps other sugary beverages, are not likely to benefit and tend to worsen renal injury associated with heat stress and dehydration.

We should state a limitation about statistics. We conducted Bonferroni correction for multiple comparisons. However, since our primary interest was whether there was worse renal injury with heat stress with rehydration with fructose compared to heat stress with water rehydration, we also performed a statistical assessment of these two groups using the t-test. We understand this method is not fair if we accounted for the effect of both heat and fructose. However, we believe that this t-test is acceptable in this case because our aim is to know only the effects of fructose intake under the heat stress condition.

\section{Conclusion}

In conclusion, rehydration with fructose in a model of heat stress and dehydration was associated with significant increased renal inflammation and a tendency for worse renal outcomes compared to equivalent rehydration with water. We recommend additional studies to investigate what concentrations of fructose and glucose may provide benefit for maintaining glucose stores while at the same time minimizing the risk for renal injury in the dehydrated patient.

\section{Abbreviations}

ACE: Angiotensin converting enzyme; ANOVA: One way analysis of variance; CKD: Chronic kidney disease; Heat + F: Heat plus fructose group; IL-

18: Interleukin 18; MCP-1: Monocyte chemoattractant protein-1; NLPR3: Nodlike receptor family-3; PAS: Periodic acid-Schiff reagent; WT: Wild type

\section{Acknowledgments}

This paper is considered a contribution from the University of Colorado Climate Change and Health Consortium.

\section{Funding}

Supported by grants from the US Department of Defense (PR130106), from Danone Research, and from the Dutch National Postcode Lottery to the Solidaridad Network and La Isla Foundation. The funding agencies provided critiques of our proposal design but had no role in the collection, analysis, interpretation, or writing of the manuscript.

\section{Availability of data and materials}

Provided upon email request to Carlos.Roncal@ucdenver.edu.

\section{Authors' contributions}

The experimental studies were performed by TM, CR and FEG. Experimental design was by TM, CR, MAL, GG, AAH, LGS, RJJ. Drafting of the paper was by 
CR, RJJ, MK, TJ, and YS, with additional editing by MAL, TI, AAH, and JG. The final version has been approved by all authors.

\section{Ethics approval}

All experiment were conducted with adherence to the NIH Guide for the Care and Use of Laboratory Animals. The animal protocol was approved by the Animal Care and Use Committee of the University of Colorado.

\section{Consent for publication}

N/A

\section{Competing interests}

Dr. Johnson, Lanaspa and Sanchez-Lozada are members of Colorado Research Partners LLC that is making inhibitors of fructose metabolism. Dr. Johnson is also on the Scientific Board of Kibow, Inc. and XORT therapeutics.

\section{Publisher's Note}

Springer Nature remains neutral with regard to jurisdictional claims in published maps and institutional affiliations.

\section{Author details}

'Division of Renal Diseases and Hypertension, Nephrology Division, Mail Stop C281, University of Colorado Anschutz Medical Campus, 12700 East 19th Ave 7th Floor Offices, Aurora, CO 80045, USA. ${ }^{2}$ Laboratory of Renal Physiopathology, Instituto Nacional de Cardiologia, Ignacio Chavez, Mexico City, Mexico. ${ }^{3}$ La Isla Network, Léon, Nicaragua.

\section{Received: 12 December 2017 Accepted: 26 June 2018}

\section{Published online: 13 July 2018}

\section{References}

1. Hunter GD. Notes on heat and "heat-stroke" at Assouan in the summer of 1886. Br Med J. 1887;2(1384):65

2. A roman experience with heat stroke in 24 B.C. Bull N Y Acad Med. 1967; 43(8):767-8.

3. Sorensen JB, Ranek L. Exertional heatstroke: survival in spite of severe hypoglycemia, liver and kidney damage. J Sports Med Phys Fitness. 1988; 28(1):108-10.

4. Brown J, Mitchell S. A complicated case of exertional heat stroke in a military setting with persistent elevation of creatine phosphokinase. Mil Med. 1992;157(2):101-3.

5. Smalley $B$, Janke RM, Cole D. Exertional heat illness in air force basic military trainees. Mil Med. 2003;168(4):298-303.

6. Casa DJ, Armstrong LE, Ganio MS, Yeargin SW. Exertional heat stroke in competitive athletes. Curr Sports Med Rep. 2005;4(6):309-17.

7. Argaud L, Ferry T, Le QH, Marfisi A, Ciorba D, Achache P, Ducluzeau R, Robert D. Short- and long-term outcomes of heatstroke following the 2003 heat wave in Lyon, France. Arch Intern Med. 2007:167(20):2177-83.

8. Kovats RS, Kristie LE. Heatwaves and public health in Europe. Eur J Pub Health. 2006;16(6):592-9.

9. Mohanaselvan A, Bhaskar E. Mortality from non-exertional heat stroke still high in India. Int J Occup Environ Med. 2014;5(4):222-4

10. Dematte JE, O'Mara K, Buescher J, Whitney CG, Forsythe S, McNamee T, Adiga RB, Ndukwu IM. Near-fatal heat stroke during the 1995 heat wave in Chicago. Ann Intern Med. 1998;129(3):173-81.

11. Rahmstorf S, Coumou D. Increase of extreme events in a warming world. Proc Natl Acad Sci U S A. 2011;108(44):17905-9.

12. Im ES, Pal JS, Eltahir EAB. Deadly heat waves projected in the densely populated agricultural regions of South Asia. Sci Adv. 2017;3(8):e1603322.

13. Kew MC, Abrahams C, Seftel HC. Chronic interstitial nephritis as a consequence of heatstroke. Q J Med. 1970;39(154):189-99.

14. Bianchi L, Ohnacker H, Beck K, Zimmerli-Ning M. Liver damage in heatstroke and its regression. A biopsy study. Hum Pathol. 1972;3(2):237-48.

15. Correa-Rotter R, Wesseling C, Johnson RJ. CKD of unknown origin in central America: the case for a Mesoamerican nephropathy. Am J Kidney Dis. 2014; 63(3):506-20.

16. Glaser J, Lemery J, Rajagopalan B, Diaz HF, Garcia-Trabanino R, Taduri G, Madero M, Amarasinghe M, Abraham G, Anutrakulchai S, et al. Climate change and the emergent epidemic of CKD from heat stress in rural communities: the case for heat stress nephropathy. Clin J Am Soc Nephrol. 2016;11(8):1472-83.
17. Johnson RJ. Pro: heat stress as a potential etiology of Mesoamerican and Sri Lankan nephropathy: a late night consult with Sherlock Holmes. Nephrol Dial Transplant. 2017;32(4):598-602.

18. Roncal Jimenez CA, Ishimoto T, Lanaspa MA, Rivard CJ, Nakagawa T, Ejaz AA, Cicerchi C, Inaba S, Le M, Miyazaki M, et al. Fructokinase activity mediates dehydration-induced renal injury. Kidney Int. 2014;86(2):294-302.

19. Cirillo P, Gersch MS, Mu W, Scherer PM, Kim KM, Gesualdo L, Henderson GN, Johnson RJ, Sautin YY. Ketohexokinase-dependent metabolism of fructose induces proinflammatory mediators in proximal tubular cells. J Am Soc Nephrol. 2009;20(3):545-53.

20. García-Arroyo FE, Cristobal M, Arellano-Buendia AS, Osorio H, Tapia E, Soto V, Madero M, Lanaspa M, Roncal-Jimenez CA, Bankir L, et al. Rehydration with soft drink-like beverages exacerbates dehydration and worsens dehydration-associated renal injury. Am J Physiol Regul Integr Comp Physiol. 2016; in press

21. Fischer RSB, Mandayam S, Chavarria D, Vangala C, Garcia MN, Garcia LL, Palma L, Garcia F, Garcia-Trabanino R, Murray KO. Clinical Evidence of Acute Mesoamerican Nephropathy. Am J Trop Med Hyg. 2017;97(4):1247-56.

22. Takahashi N, Boysen G, Li F, Li Y, Swenberg JA. Tandem mass spectrometry measurements of creatinine in mouse plasma and urine for determining glomerular filtration rate. Kidney Int. 2007;71(3):266-71.

23. Garcia-Arroyo FE, Cristobal M, Arellano-Buendia AS, Osorio H, Tapia E, Soto V, Madero M, Lanaspa MA, Roncal-Jimenez C, Bankir L, et al. Rehydration with soft drink-like beverages exacerbates dehydration and worsens dehydration-associated renal injury. Am J Physiol Regul Integr Comp Physiol. 2016:311(1):R57-65.

24. Garcia-Arroyo FE, Tapia E, Blas-Marron MG, Gonzaga G, Silverio O, Cristobal M, Osorio H, Arellano-Buendia AS, Zazueta C, Aparicio-Trejo OE, et al. Vasopressin mediates the renal damage induced by limited fructose rehydration in recurrently dehydrated rats. Int J Biol Sci. 2017;13(8):961-75.

25. Wolf JP, Nguyen NU, Dumoulin G, Berthelay S. Influence of hypertonic monosaccharide infusions on the release of plasma arginine vasopressin in normal humans. Horm Metab Res. 1992;24(8):379-83.

26. Song Z, Roncal-Jimenez CA, Lanaspa-Garcia MA, Oppelt SA, Kuwabara M, Jensen T, Milagres T, Andres-Hernando A, Ishimoto T, Garcia GE, et al. Role of fructose and fructokinase in acute dehydration-induced vasopressin gene expression and secretion in mice. J Neurophysiol. 2017:117(2):646-54.

27. Roncal CA, Mu W, Croker B, Reungjui S, Ouyang X, Tabah-Fisch I, Johnson RJ, Ejaz AA. Effect of elevated serum uric acid on cisplatin-induced acute renal failure. Am J Physiol Renal Physiol. 2007;292(1):F116-22.

28. Xiao J, Zhang XL, Fu C, Han R, Chen W, Lu Y, Ye Z. Soluble uric acid increases NALP3 inflammasome and interleukin-1beta expression in human primary renal proximal tubule epithelial cells through the toll-like receptor 4-mediated pathway. Int J Mol Med. 2015;35(5):1347-54.

29. O'Donnell JK, Tobey M, Weiner DE, Stevens LA, Johnson S, Stringham P, Cohen B, Brooks DR. Prevalence of and risk factors for chronic kidney disease in rural Nicaragua. Nephrol Dial Transplant. 2011;26(9):2798-805.

30. Ramirez-Rubio O, McClean MD, Amador JJ, Brooks DR. An epidemic of chronic kidney disease in central America: an overview. J Epidemiol Community Health. 2013:67(1):1-3.

31. Wesseling C, Crowe J, Hogstedt C, Jakobsson K, Lucas R, Wegman D. Mesoamerican nephropathy: report from the first international research workshop on MeN. Heredia, Costa Rica: SALTRA / IRET-UNA; 2013. https:// issuu.com/apacomunicaciones/docs/report_from_the_first_international.

32. Garcia-Trabanino R, Jarquin E, Wesseling C, Johnson RJ, Gonzalez-Quiroz M, Weiss I, Glaser J, Jose Vindell J, Stockfelt L, Roncal C, et al. Heat stress, dehydration, and kidney function in sugarcane cutters in El Salvador-a cross-shift study of workers at risk of Mesoamerican nephropathy. Environ Res. 2015:142:746-55.

33. Wijkstrom J, Leiva R, Elinder CG, Leiva S, Trujillo Z, Trujillo L, Soderberg M, Hultenby K, Wernerson A. Clinical and pathological characterization of mesoamerican nephropathy: a new kidney disease in central america. Am J Kidney Dis. 2013;62(5):908-18.

34. Roncal-Jimenez CA, Milagres T, Andres-Hernando A, Kuwabara M, Jensen T, Song Z, Bjornstad P, Garcia GE, Sato Y, Sanchez-Lozada LG, et al. Effects of exogenous desmopressin on a model of heat stress nephropathy in mice. Am J Physiol Renal Physiol. 2017;312(3):F418-26.

35. Bankir L, Bouby N, Ritz E. Vasopressin: a novel target for the prevention and retardation of kidney disease? Nat Rev Nephrol. 2013;9(4):223-39. 